\title{
Long-term video EEG monitoring for diagnosis of psychogenic nonepileptic seizures
}

\author{
This article was published in the following Dove Press journal: \\ Neuropsychiatric Disease and Treatment \\ 15 October 2014 \\ Number of times this article has been viewed
}

\author{
Evan R Gedzelman \\ Suzette M LaRoche \\ Department of Neurology, Emory \\ Epilepsy Program, Atlanta, GA, USA
}

Correspondence: Evan R Gedzelman; Suzette M LaRoche

Department of Neurology, Emory

Epilepsy Program, I365A Clifton Road

NE, Atlanta, GA, 30322, USA

$\mathrm{Tel}+\mathrm{I} 4047783444$

Fax +I 4047784216

Email evan.r.gedzelman@emory.edu; slaroch@emory.edu

\begin{abstract}
Psychogenic nonepileptic seizures have long been known by many names. A short list includes hysteroepilepsy, hysterical seizures, pseudoseizures, nonepileptic events, nonepileptic spells, nonepileptic seizures, and psychogenic nonepileptic attacks. These events are typically misdiagnosed for years and are frequently treated as electrographic seizures and epilepsy. These patients experience all the side effects of antiepileptic drugs and none of the benefits. Video electroencephalogram (EEG) monitoring is the gold standard diagnostic test that can make a clear distinction between psychogenic nonepileptic seizures and epilepsy. Video EEG allows us to correctly characterize the patient's events and therefore properly diagnose and direct management. As a result, years of faulty management and wasted health care dollars can be avoided.
\end{abstract}

Keywords: psychogenic nonepileptic seizures, electroencephalogram, video EEG monitoring, conversion disorder, cognitive behavioral therapy, antiepileptic drugs

\section{Introduction}

Unlike epileptic seizures, psychogenic nonepileptic seizures (PNES) do not result from abnormal electrical discharges from the brain, but rather are a physical manifestation of a psychological disturbance. They fall under the broader category of somatoform disorders and are classified as conversion disorder based on the Diagnostic and Statistical Manual of Mental Disorders, 4th edition. These events are involuntary and out of the patient's conscious control. Rarely, PNES can occur secondary to malingering or factitious disorder, in which case the behavior is purposeful with intent for primary or secondary gain. Making this diagnosis is fraught with medicolegal hazards and is hard to prove. ${ }^{1}$ PNES should also be differentiated from physiological, nonepileptic events such as syncope, cataplexy, migraine, paroxysmal movement disorders, breathholding spells, and shuddering attacks in children. ${ }^{1}$ The semiology of the event can help distinguish PNES from epilepsy. Table 1 summarizes pertinent semiology and timing features that can assist in differentiating between PNES and epileptic seizures. ${ }^{2}$ The importance of recognizing these semiological characteristics will be discussed in further detail later in this paper.

PNES is commonly misdiagnosed as epilepsy, and patients are often treated for years with an incorrect diagnosis. ${ }^{3}$ It is a common indication for referral to epilepsy centers, where approximately $30 \%$ of epilepsy monitoring unit (EMU) admissions for refractory epilepsy are appropriately diagnosed with PNES. ${ }^{3}$ In fact, it is the most frequent nonepileptic condition seen at epilepsy centers, more common than physiological nonepileptic conditions. ${ }^{3}$ In addition, of the $1 \%$ of the US population with epilepsy, $5 \%-20 \%$ also experience PNES. ${ }^{4}$ 
Table I Differences in semiology and timing between PNES and epileptic seizures

\begin{tabular}{lll}
\hline & PNES & Epileptic seizure \\
\hline Timing & & \\
Directly induced by stress or & + & + \\
confrontation & & \\
Waxes and wanes & ++ & - \\
Occurs in physician office & ++ & - \\
Worsens with witnesses in the & ++ & - \\
room & & \\
Semiology & & \\
Crying & + & - \\
Whispering & + & - \\
Stuttering & + & - \\
Opisthotonic posture & + & - \\
Full body shaking with & ++ & - \\
preserved awareness & & \\
Ictal cry & - & ++ (before GTC seizure) \\
Post-ictal buzz saw snore & - & ++ (after GTC seizure) \\
Eyes closed & ++ & - \\
Head/body/eye version & - & ++ (focal seizure) \\
Fencer's posture & - & ++ (focal seizure) \\
Side-to-side head movement & + & - \\
Pelvic thrusting & + & + \\
Cyanosis & - & + (during GTC seizure) \\
Severe injury or trauma (burns & - & + (during GTC seizure) \\
and fractures) & & \\
\hline Notes & & \\
\hline
\end{tabular}

Notes: + represents "frequent", + represents "occasional", and - represents "absent." Abbreviations: PNES, psychogenic nonepileptic seizures; GTC, generalized tonic-clonic.

Over time, the tangible and intangible costs add up for the patient, the medical system, and society. The individual with PNES has an estimated US $\$ 100,000$ lifetime cost for diagnostic tests, procedures, and medications. ${ }^{3}$ In the US alone, up to US $\$ 900$ million per year is unnecessarily spent on diagnostic evaluations, repeated labs, antiepileptic drugs (AEDs), and emergency department utilization for the evaluation and management of patients with PNES. ${ }^{5}$

PNES occur more frequently in women than men (accounting for $80 \%$ of all cases) and the majority of patients are $15-35$ years of age $(83 \%))^{3}$ However, some recent studies suggest that this condition might be more prevalent than was previously thought in men and in the elderly. ${ }^{6}$ Based on one study, approximately $30 \%$ of patients undergoing video electroencephalogram (EEG) monitoring in both the civilian and Veterans Administration populations (mostly male and of older age) were diagnosed with PNES. ${ }^{6}$ PNES has also been observed in diverse ethnic groups. ${ }^{7}$

With an average delay to diagnosis of 7-10 years, it is clear that the index of suspicion for PNES is not high enough when evaluating patients with refractory seizures. ${ }^{8}$ Additionally, many physicians are unaware that a patient can have both epilepsy and PNES. Of note, one study reported that $10 \%$ of patients diagnosed with PNES also had epileptic seizures. ${ }^{9}$ Therefore, when patients with multiple event types undergo EEG monitoring, additional care must be taken to confirm that all of the patients' typical events have been captured and appropriately categorized. Otherwise, neither PNES nor epilepsy can be absolutely excluded.

The health risk of persons with PNES misdiagnosed with epilepsy is considerable. Aggressive treatment to stop the seizure sometimes results in oversedation, requiring paralysis and intubation. In one study in a pediatric population, ${ }^{10}$ $5 \%-20 \%$ of patients with presumed status epilepticus were eventually determined to be in "pseudo-status" and, therefore, could have avoided the dangers of paralysis and intubation if properly diagnosed. Other risks are posed by toxic medication levels, the insertion of central catheter lines, venous cutdowns, vagal nerve stimulator placement, and evaluations for temporal lobectomy. ${ }^{5}$ Vocational costs add to the burden. The $50 \%$ disability rate for persons with PNES equals that of patients with epilepsy. ${ }^{4}$ All these factors underscore the need for an accurate diagnosis early in the course, opening the door to appropriate treatments. Furthermore, delay to diagnosis worsens the prognosis for PNES. ${ }^{11}$

Even though PNES are very common, there are limited data regarding outcomes. A few studies ${ }^{12,13}$ have looked at event remission after the diagnosis of PNES is made with video EEG monitoring. At follow-up, approximately $30 \%$ of patients were shown to be event free. However, these studies are small and do not take into account whether the patients receive ongoing therapy such as cognitive behavioral therapy $(\mathrm{CBT}) .^{12,13}$

\section{Video EEG monitoring background}

Video EEG is the gold standard for the diagnosis of PNES. It simultaneously records the patient's brain electrical activity and captures corresponding behaviors on video. This testing is indicated in all patients with frequent paroxysmal events that persist despite taking antiseizure medications. In most cases, the treating epileptologist can differentiate between PNES and epileptic seizures using video analysis alone. However, combined electroclinical analysis of the clinical semiology or clinical pattern of the event and the ictal EEG findings allows for a definitive diagnosis in nearly all cases. ${ }^{14}$

No laboratory tests or imaging studies are as reliable as video EEG monitoring at identifying and characterizing PNES, and differentiating them from epileptic seizures. However, elevated serum prolactin may differentiate generalized tonic-clonic seizures from PNES. ${ }^{15}$ Serum prolactin rise within 30 minutes of ictus onset may be helpful in 
differentiating generalized tonic-clonic seizures from PNES, but at best is useful as an adjunctive diagnostic test to the delineation of PNES from epileptic seizures, particularly since the absence of any change in prolactin level cannot exclude the possibility of an epileptic seizure. ${ }^{2}$ Structural neuroimaging abnormalities can neither confirm nor exclude either disorder since PNES may occur in the presence of focal lesions such as mesial temporal sclerosis. ${ }^{16}$

A glaring deficiency of routine and/or ambulatory EEG compared to the gold standard of continuous video EEG monitoring is the absence of video to correlate with the EEG findings. Background EEG findings can be misleading when trying to distinguish epileptic seizures from PNES, particularly when a typical event is not captured. For example, $30 \%$ of patients with epilepsy have normal EEGs at presentation. ${ }^{17}$ On the other hand, there are nonspecific EEG abnormalities or benign variants (subtly different than normal waveforms, but not pathological) that can be seen in more than $10 \%$ of healthy individuals. ${ }^{17}$ Frequently, these normal variants are read as pathological and can contribute to erroneous diagnosis in patients with PNES. In addition, one large retrospective study showed that approximately $0.5 \%$ of healthy individuals with neither a diagnosis of epilepsy nor PNES had "unequivocal" epileptiform discharges without any previous documented pathology or history of seizures. ${ }^{18}$

\section{Clinical history}

When admitting patients to the EMU, special care must be taken to procure an in-depth history. This could give the necessary information that leads to the suspicion of PNES versus epilepsy. A lack of efficacy of AEDs may be the first sign that the events could be psychogenic. Like other somatoform disorders, patients with PNES often demonstrate features of suggestibility, such as the reproduction of symptoms upon the physician's examination. Risk factors for PNES may include a history of physical and sexual abuse, and a floridly positive review of systems. ${ }^{19}$ About three-quarters of patients report prior emotional or physical trauma such as abuse, bereavement, serious illness, accidents, or assault. ${ }^{19}$ Chronic pain or a history of fibromyalgia are often associated with psychogenic symptoms, and their presence can have a high predictive value for PNES of 70\%-80\%. ${ }^{1,19}$

\section{Semiology}

Analysis of the ictal semiology (ie, video) is of paramount importance. Certain characteristics of motor phenomena are strongly associated with PNES: gradual onset or termination; occurrence of events during "pseudosleep"; discontinuous (stop-and-go) movements; irregular or asynchronous (out-of-phase) activity; side-to-side head movement; pelvic thrusting; opisthotonic posturing; stuttering; weeping; preserved awareness during bilateral motor activity; and postictal whispering. ${ }^{1}$

Additional evaluation focusing on ocular and facial movements can help further distinguish between PNES and epilepsy. ${ }^{2,13}$ Eye closure, especially of long duration, is highly associated with PNES. Patients with PNES may also show geotropic eye movements (turning or movement of the eyes in the direction of gravity). Ictal stuttering and postictal whispering are also suggestive of PNES. ${ }^{2,13}$ On the other hand, there are features that can be very suggestive of epileptic seizures and that are not typically seen in PNES, such as postictal nose rubbing and cough, which are common in temporal lobe epilepsy, and stertorous breathing in the postictal period following an epileptic generalized tonic-clonic seizure. Stereotypy strongly suggests epileptic seizures rather than PNES. ${ }^{1}$

Pelvic thrusting can be seen in both epilepsy (especially frontal lobe seizures) and PNES. Out-of-phase, or asynchronous, movements tend to favor PNES, especially if the movements wax and wane over many minutes and do not occur during sleep. In contrast, frontal lobe seizures typically arise from sleep, involve vocalization, as well as quick and tonic posturing or bicycling movements of the legs, and are brief. ${ }^{2}$ Whether associated physical injuries can differentiate between PNES and epileptic seizures is a matter of debate. Some sources conclude that physical injuries can occur as a result of either epileptic seizures or PNES. ${ }^{13}$ However, in our experience, objective injuries such as tongue bite and bone fracture are seen more frequently with epilepsy and subjective injury is often over-reported and exaggerated in the PNES population.

In a study of 120 seizures from 35 patients (36 PNES and 84 epileptic seizures), only a few clinical signs were reliable in predicting a diagnosis of PNES versus epilepsy. ${ }^{20}$ Useful clues to the diagnosis of PNES included preserved awareness during bilateral motor activity, as unresponsiveness is almost always present during bilateral motor activity in an epileptic seizure. PNES were also predicted by eye flutter, as well as by events affected by the presence of bystanders (intensified or alleviated). Epileptic seizures were predicted by abrupt onset, eye opening/widening, and postictal confusion or sleep. Importantly, compared to an expert review of these signs on video recording, eyewitness reports of these symptoms were not reliable. ${ }^{20}$ 
The categorization of PNES has been a difficult task in the past, as the semiology is varied. A few have made attempts at categorizations based on retrospective cluster analysis. ${ }^{21}$ One study divided PNES into six stereotypic categories (utilizing video EEG): 1) rhythmic motor PNES; 2) hypermotor PNES characterized by violent movements; 3) complex motor PNES; 4) dialeptic PNES characterized by unresponsiveness without motor manifestations; 5) nonepileptic auras characterized by subjective sensations without external manifestations; and 6) mixed PNES with combinations of the aforementioned categories. ${ }^{21}$

Another study ${ }^{22}$ conducted multiple correspondence analysis and hierarchical cluster analysis based on a review of video EEG studies to construct a practical and useful semiological classification of PNES. This study separated the patterns into five clusters of signs: 1) dystonic attack with primitive gestural activity; 2) paucikinetic attack with preserved responsiveness; 3) pseudosyncope; 4) hyperkinetic prolonged attack with hyperventilation and auras; and 5) axial dystonic prolonged attack. ${ }^{22}$ The categorization of PNES has potential implications for diagnosis and prognosis, with a number of studies indicating that the outcome of PNES may vary among different types. ${ }^{13,23}$

\section{EEG findings}

The diagnosis of PNES is made conclusively by coregistering video with EEG findings. While coregistered EEG findings can confirm a nonepileptic event origin, the differentiation between psychogenic versus physiologic etiology relies upon careful evaluations of the patient's clinical history, event semiology, physical exam, psychosocial morbidities, and/or psychometric testing. ${ }^{2}$ Typically in PNES, the EEG background is normal in the waking state prior to the ictus, during the ictus, and after the ictus (see Figures 1-3).

Uninterpretable EEG recordings may occur if movement generates excessive artifact that obscures the EEG background. However, in these cases, completely normal EEG background immediately preceding and following a typical "convulsive event" is highly suggestive of PNES. ${ }^{1}$ The ictal EEG can also be limited in some focal seizure types, as the EEG background may show minimal or no change. Focal seizures without clear alteration of awareness are the most

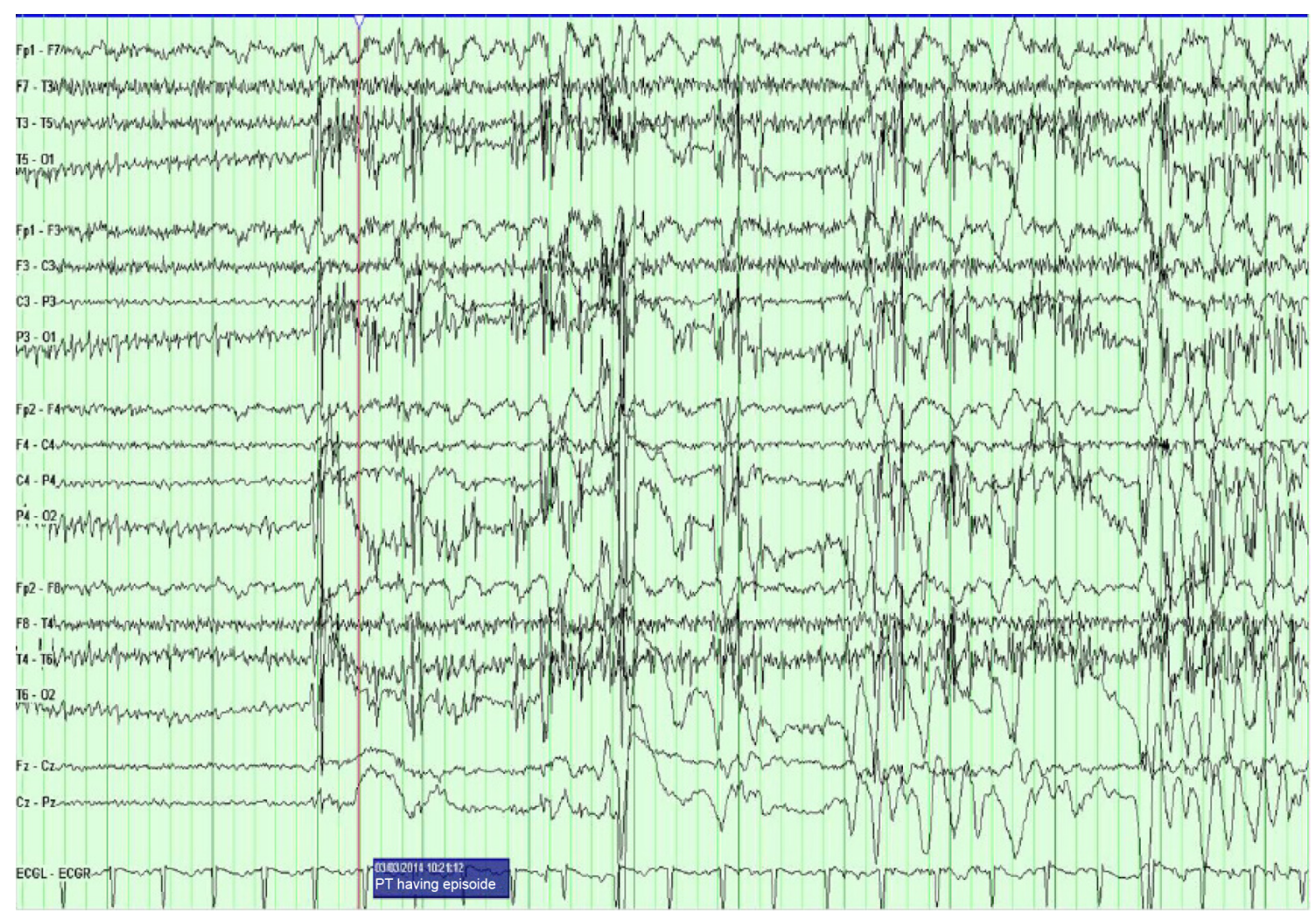

Figure I The PT transitions into a typical PNES.

Notes: Immediately prior to it, the EEG background shows a normal waking rhythm. The EEG is then obscured by movement and muscle artifact that is most prominent in the posterior regions.

Abbreviations: PT, patient; PNES, psychogenic nonepileptic seizure; EEG, electroencephalogram. 


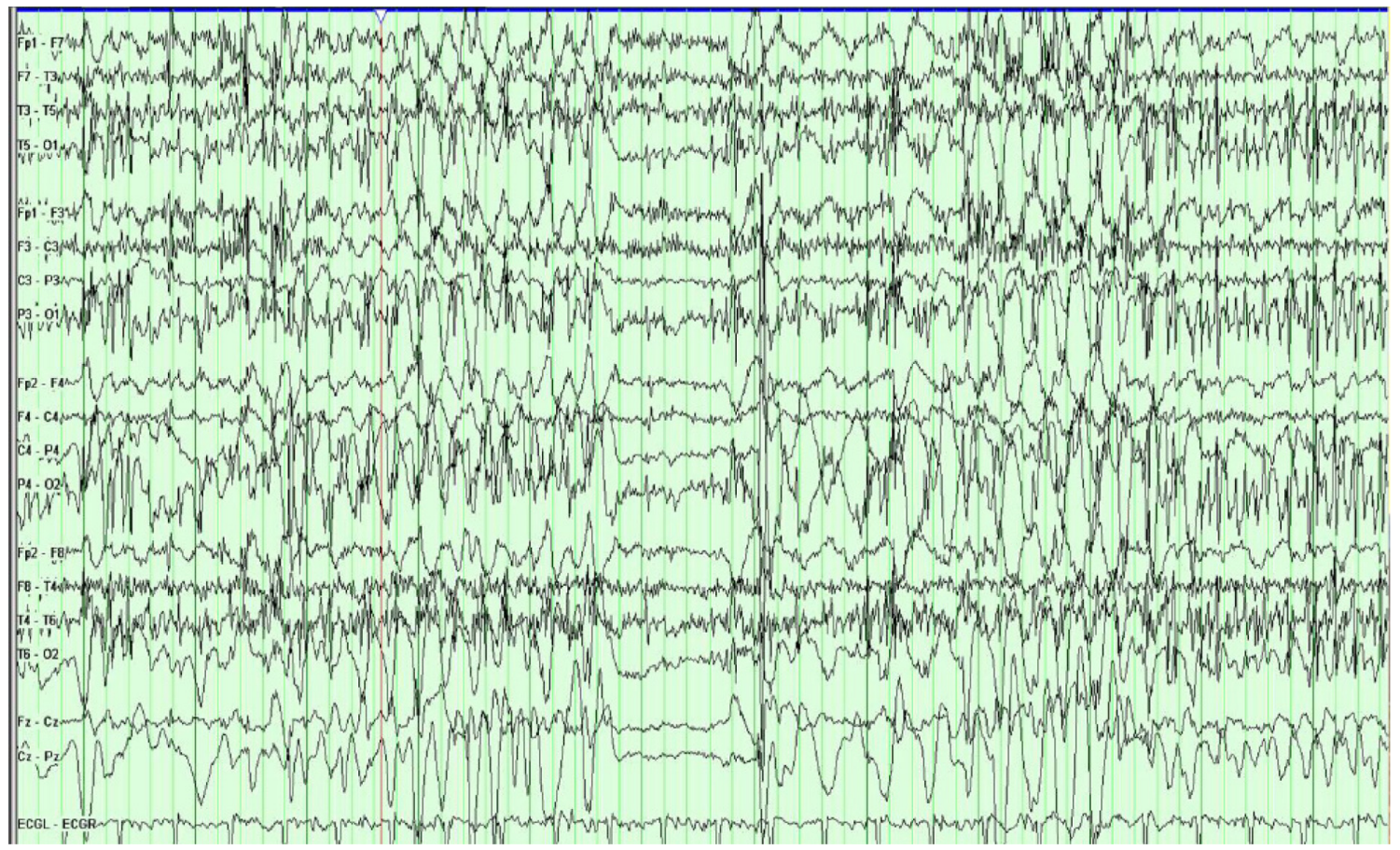

Figure 2 The patient is in the middle of a typical PNES.

Notes: The EEG background is obscured by movement and muscle artifact that is most prominent in the posterior regions. There is an embedded I-second period when the movement stops and normal background is seen before the background is again obscured by movement and muscle artifact.

Abbreviations: PNES, psychogenic nonepileptic seizure; EEG, electroencephalogram.

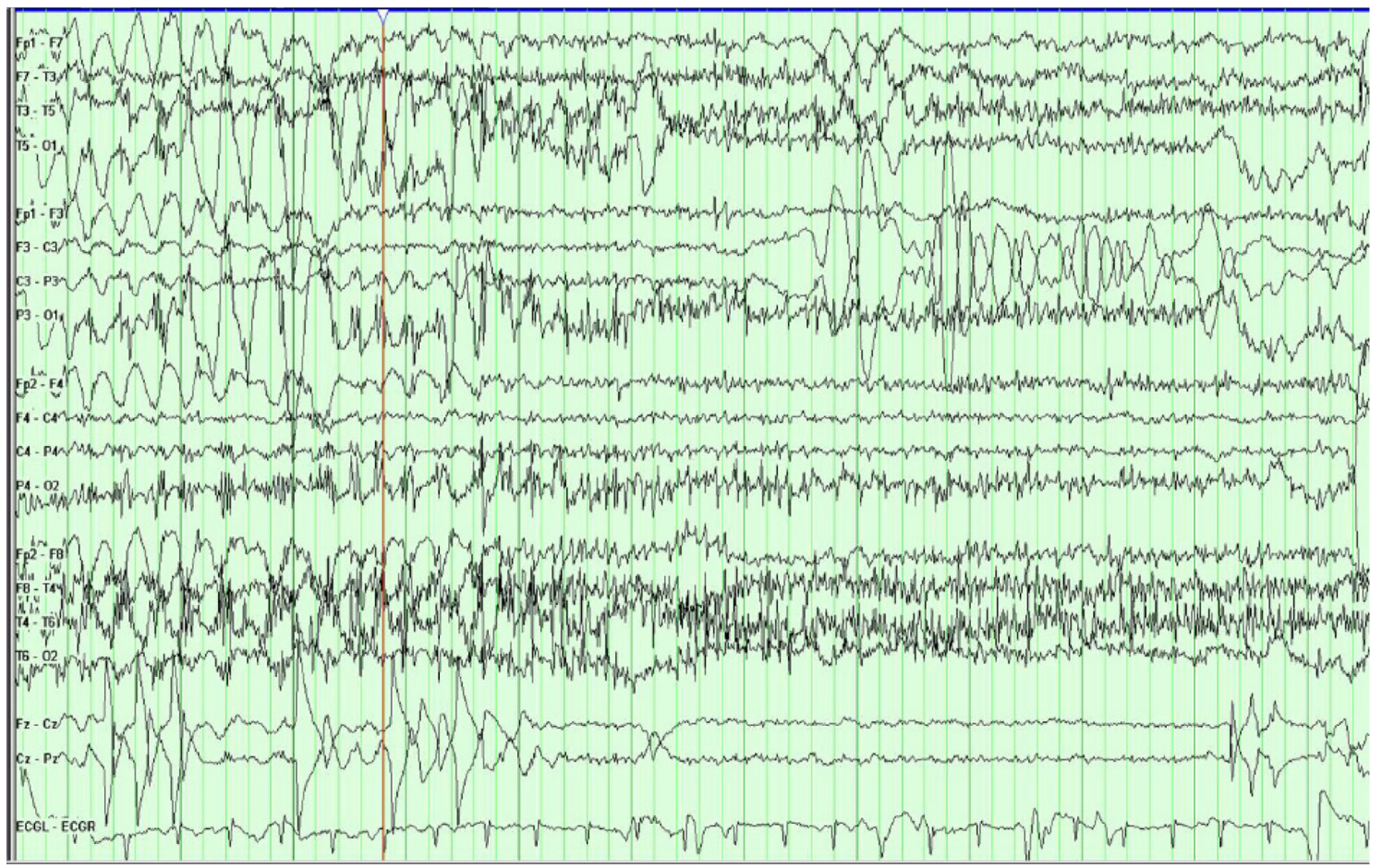

Figure 3 The patient transitions out of a typical PNES.

Notes: Initially, the slide shows EEG background obscured by movement and muscle artifact that is most prominent in the posterior regions. Immediately after it, the EEG background returns to a normal waking background.

Abbreviations: PNES, psychogenic nonepileptic seizure; EEG, electroencephalogram. 
common type of seizure that can be unaccompanied by significant EEG changes in approximately $30 \%$ of cases. ${ }^{1}$ Seizures that occur without impairment of awareness involve sensory phenomena such as paresthesias, psychiatric symptoms of olfactory hallucinations, fear and déjà vu, focal motor or brief tonic movements, and autonomic symptoms. These are typically brief and can be of frontal or temporal lobe origin. ${ }^{1}$

\section{Seizure induction}

What can be done in the cases when the typical event has not been captured during the designated monitoring period in the EMU? Provocative techniques including activation procedures and seizure "induction" can be very helpful. When combined with ictal video EEG monitoring, these techniques have a very high specificity for PNES. ${ }^{24}$ However, provocative induction is only helpful when the reproduced event is confirmed by the patient or his/her family to be strongly characteristic of the habitual event of interest. Otherwise, due to the anxious and suggestible nature of some patients, provocative induction can frequently trigger nonspecific and nonrelevant symptoms. ${ }^{24}$ Currently accepted techniques include photic stimulation, hyperventilation, and verbal suggestion. One advantage of photic stimulation and hyperventilation is that these maneuvers are used during routine EEG as well, and therefore do not appear out of the ordinary to the patient. Even without using placebo, provocative induction with photic stimulation, verbal suggestion, and hyperventilation can achieve up to an $84 \%$ success rate in capturing typical events. ${ }^{25}$ An intravenous injection of saline is used in some centers, but is controversial because of ethical concerns. $^{25}$ The principle of suggestibility, a characteristic of all somatoform disorders, is the crucial component to all provocative techniques.

\section{Delivering the diagnosis}

After the diagnosis of PNES is definitively made, the question then arises as to how to approach the patient with this new and unfamiliar diagnosis. It is extremely helpful for discussions of expectations and the possibility of PNES to take place during the patient's outpatient clinic visit, prior to admission for video EEG monitoring. Taking this step opens a dialogue of trust and transparency that will likely allow the patient to accept the diagnosis more readily when the final diagnosis is made.

The role of the epileptologist is of paramount importance in delivering the diagnosis of PNES. The initial explanation in the EMU after confirmation is probably the most important step in initiating treatment. ${ }^{11}$ Patients and families are unlikely to comply with recommendations unless they understand and accept the diagnosis. A negative reaction to the explanation can affect outcomes. Therefore, the epileptologist must explain the diagnosis with unambiguous terms (for example, "stress-induced") while simultaneously remaining compassionate, firm, and confident. The diagnosis of PNES must be clearly delineated from a diagnosis of epileptic seizures. Because this delivery of diagnosis is so important, many providers follow a templated script such as the protocol by Shen et al. ${ }^{26}$ This protocol emphasizes the goal of conveying to the patient the importance of knowing the nonepileptic nature of the spells and the need for psychiatric follow-up. It also allows for elicitation of the patient's sexual abuse history and the use of suggestion to aid in the control of PNES. When the PNES diagnosis is properly conveyed and then accepted by the patient, then up to $\sim 30 \%$ of the patients enjoy remission of PNES, often without any further intervention. ${ }^{27}$ This concept further underscores the importance of a proper delivery of the PNES diagnosis by the epileptologist.

The epileptologist can continue to be involved afterwards and can help with weaning AEDs, though the psychological treatment should be addressed by the psychologist or psychiatrist. AEDs do not cure and do not typically help PNES, and AED toxicity may worsen this disorder. ${ }^{28}$ A study that monitored the discontinuation of AEDs in 78 patients with PNES revealed that the majority of these patients experienced a reduction in the frequency of PNES when taken off the medication, indicating that withdrawal of AED treatment is not only safe but can also improve outcomes. ${ }^{29}$

The issues of driving and disability should be addressed after the diagnosis of PNES is made. Unfortunately, there is little data regarding PNES and driving safety; however, no evidence exists, which demonstrates that patients with PNES have an increased risk of car accidents. Physicians tend to take an experiential approach with this. Some may restrict driving in all PNES patients, while others approach this restriction on a case-by-case basis. ${ }^{30}$ Since PNES is a psychiatric diagnosis that requires treatment by mental health professionals, disability should ideally be determined by the treating mental health professionals.

Securing follow-up with mental health professionals is of paramount importance in patients with video EEG-confirmed PNES. Unfortunately, these services are not always readily available. Additionally, there are obstacles to treatment, including mental health providers that are not familiar with PNES and its treatment. Providing a video recording of the patient's typical event can be a useful method to educate and reinforce the diagnosis with the mental health team. ${ }^{31}$ 


\section{Treatment}

There is mounting evidence that CBT is the treatment of choice for PNES. Goldstein et $\mathrm{al}^{32}$ reported that CBT significantly reduced event activity in patients with PNES compared with standard medical care. Another prospective trial evaluated the effect of CBT on the reduction of PNES events. ${ }^{33}$ Subjects with video EEG-confirmed PNES were treated with CBT in 12 weekly outpatient sessions. Seventeen of 21 patients completed the CBT intervention, and eleven of the 17 completers reported no seizures by the final CBT session. Mean scores on scales of depression, anxiety, somatic symptoms, quality of life, and psychosocial functioning showed improvement from baseline. This study demonstrates that CBT reduces PNES events and improves psychiatric symptoms, psychosocial functioning, and quality of life. ${ }^{33}$

There is also growing evidence that selective serotonin reuptake inhibitor medications may play a role in modifying the depression that is often seen concurrently with PNES. ${ }^{34,35}$ Additionally, selective serotonin reuptake inhibitors may play a role in reducing the frequency of events in patients with PNES. However, this must be taken with a degree of skepticism, as larger scale studies are needed with higher levels evidence. ${ }^{34,35}$

\section{Conclusion}

PNES is a disorder that affects a significant percentage of patients with presumed epileptic seizures. On average, it takes 7-10 years to arrive at a definitive diagnosis of PNES, suggesting that the index of suspicion is too low and that misdiagnosis is commonplace. ${ }^{4}$ This leads to years of inappropriate treatment, during which time patients may be consigned to take multiple AEDs and suffer a myriad of untoward side effects while deriving none of the purported benefits of these medications. A proper diagnosis is absolutely key to appropriate treatment. Video EEG is a unique tool that is the gold standard for the diagnosis of PNES. There is no better test for arriving at a near certain diagnosis. Once the diagnosis has been securely established, the appropriate delivery and explanation of the disorder must be given to the patient. The prognosis relies heavily on the clarity of this explanation and on whether the patient receives adequate follow-up from mental health providers. Although the current standard management of PNES includes CBT, there is still much to be learned regarding optimum treatment strategies and outcomes.

\section{Disclosure}

The authors report no conflicts of interest in this work.

\section{References}

1. Benbadis SR. Nonepileptic behavioral disorders: diagnosis and treatment. Continuum (Minneap Minn). 2013;19(3 Epilepsy):715-729.

2. LaFrance WC Jr. Psychogenic nonepileptic seizures. Curr Opin Neurol. 2008;21(2):195-201.

3. Benbadis SR, O'Neill E, Tatum WO, Heriaud L. Outcome of prolonged video-EEG monitoring at a typical referral epilepsy center. Epilepsia. 2004;45(9):1150-1153.

4. LaFrance WC Jr, Benbadis SR. Avoiding the costs of unrecognized psychological nonepileptic seizures. Neurology. 2006;66(11):1620-1621.

5. Martin RC, Gilliam FG, Kilgore M, Faught E, Kuzniecky R. Improved health care resource utilization following video-EEG-confirmed diagnosis of nonepileptic psychogenic seizures. Seizure. 1998;7(5):385-390.

6. Salinsky M, Spencer D, Boudreau E, Ferguson F. Psychogenic nonepileptic seizures in US veterans. Neurology. 2011;77(10):945-950.

7. Devinsky O, Gazzola D, LaFrance WC. Differentiating between nonepileptic and epileptic seizures. Nat Rev Neurol. 2011;7(4):210-220.

8. Reuber M, Fernández G, Bauer J, Helmstaedter C, Elger CE. Diagnostic delay in psychogenic nonepileptic seizures. Neurology. 2002;58(3) 493-495.

9. Benbadis SR, Agrawal V, Tatum WO. How many patients with psychogenic nonepileptic seizures also have epilepsy? Neurology. 2001;57(5): 915-917.

10. Pakalnis A, Paolicchi J, Gilles E. Psychogenic status epilepticus in children: psychiatric and other risk factors. Neurology. 2000;54(4):969-970.

11. Carton S, Thompson PJ, Duncan JS. Non-epileptic seizures: patients' understanding and reaction to the diagnosis and impact on outcome. Seizure. 2003;12(5):287-294.

12. Arain AM, Hamadani AM, Islam S, Abou-Khalil BW. Predictors of early seizure remission after diagnosis of psychogenic nonepileptic seizures. Epilepsy Behav. 2007;11(3):409-412.

13. Reuber M, Pukrop R, Bauer J, Helmstaedter C, Tessendorf N, Elger CE. Outcome in psychogenic nonepileptic seizures: 1 to 10-year follow-up in 164 patients. Ann Neurol. 2003;53(3):305-311.

14. Avbersek A, Sisodiya S. Does the primary literature provide support for clinical signs used to distinguish psychogenic nonepileptic seizures from epileptic seizures? J Neurol Neurosurg Psychiatry. 2010;81(7): 719-725.

15. Chen DK, So YT, Fisher RS; Therapeutics and Technology Assessment Subcommittee of the American Academy of Neurology. Use of serum prolactin in diagnosing epileptic seizures: report of the Therapeutics and Technology Assessment Subcommittee of the American Academy of Neurology. Neurology. 2005;65(5):668-675.

16. Lowe MR, De Toledo JC, Rabinstein AA, Giulla MF. MRI evidence of mesial temporal sclerosis in patients with psychogenic nonepileptic seizures. Neurology. 2001;56(6):823.

17. Mellers JD. The approach to patients with "non-epileptic seizures". Postgrad Med J. 2005;81(958):498-504.

18. Gregory RP, Oates T, Merry RT. Electroencephalogram epileptiform abnormalities in candidates for aircrew training. Electroencephalogr Clin Neurophysiol. 1993;86(1):75-77.

19. Benbadis SR. A spell in the epilepsy clinic and a history of "chronic pain" or "fibromyalgia" independently predict a diagnosis of psychogenic seizures. Epilepsy Behav. 2005;6(2):264-265.

20. Syed TU, LaFrance WC Jr, Kahriman ES, et al. Can semiology predict psychogenic nonepileptic seizures? A prospective study. Ann Neurol. 2011;69(6):997-1004.

21. Seneviratne U, Reutens D, D’Souza W. Stereotypy of psychogenic nonepileptic seizures: insights from video-EEG monitoring. Epilepsia. 2010;51(7):1159-1168.

22. Hubsch C, Baumann C, Hingray C, et al. Clinical classification of psychogenic non-epileptic seizures based on video-EEG analysis and automatic clustering. J Neurol Neurosurg Psychiatry. 2011;82(9): 955-960.

23. Selwa LM, Geyer J, Nikakhtar N, Brown MB, Schuh LA, Drury I. Nonepileptic seizure outcome varies by type of spell and duration of illness. Epilepsia. 2000;41(10):1330-1334. 
24. Lancman ME, Asconapé JJ, Craven WJ, Howard G, Penry JK. Predictive value of induction of psychogenic seizures by suggestion. Ann Neurol. 1994;35(3):359-361.

25. Benbadis SR, Johnson K, Anthony K, et al. Induction of psychogenic nonepileptic seizures without placebo. Neurology. 2000;55(12):1904-1905.

26. Shen W, Bowman ES, Markand ON. Presenting the diagnosis of pseudoseizure. Neurology. 1990;40(5):756-759.

27. McKenzie P, Oto M, Russell A, Pelosi A, Duncan R. Early outcomes and predictors in 260 patients with psychogenic nonepileptic attacks. Neurology. 2010;74(1):64-69.

28. Niedermeyer E, Blumer D, Holscher E, Walker BA. Classical hysterical seizures facilitated by anticonvulsant toxicity. Psychiatr Clin (Basel). 1970;3(2):71-84.

29. Oto M, Espie C, Pelosi A, Selkirk M, Duncan R. The safety of antiepileptic drug withdrawal in patients with non-epileptic seizures. J Neurol Neurosurg Psychiatry. 2005;76(12):1682-1685.
30. Benbadis SR, Blustein JN, Sunstad L. Should patients with psychogenic nonepileptic seizures be allowed to drive? Epilepsia. 2000;41(7): 895-897.

31. Benbadis SR. The problem of psychogenic symptoms: is the psychiatric community in denial? Epilepsy Behav. 2005;6(1):9-14.

32. Goldstein LH, Chalder T, Chigwedere C, et al. Cognitive-behavioral therapy for psychogenic nonepileptic seizures: a pilot RCT. Neurology. 2010;74(24):1986-1994.

33. LaFrance WC, Miller IW, Ryan CE, et al. Cognitive behavioral therapy for psychogenic nonepileptic seizures. Epilepsy Behav. 2009;14(4): 591-596.

34. LaFrance WC, Keitner GI, Papandonatos GD, et al. Pilot pharmacologic randomized controlled trial for psychogenic nonepileptic seizures. Neurology. 2010;75(13):1166-1173.

35. LaFrance WC, Syc S. Depression and symptoms affect quality of life in psychogenic nonepileptic seizures. Neurology. 2009;73(5):366-371.

\section{Publish your work in this journal}

Neuropsychiatric Disease and Treatment is an international, peerreviewed journal of clinical therapeutics and pharmacology focusing on concise rapid reporting of clinical or pre-clinical studies on a range of neuropsychiatric and neurological disorders. This journal is indexed on PubMed Central, the 'PsycINFO' database and CAS, and is the official journal of The International Neuropsychiatric Association (INA). The manuscript management system is completely online and includes a very quick and fair peer-review system, which is all easy to use. Visit http://www.dovepress.com/testimonials.php to read real quotes from published authors. 